\title{
Taking a deeper dive into MPI: There's more there than meets the eye
}

\author{
Steven Port, $M D^{\mathrm{a}}$ \\ a Advocate Aurora Health, Aurora Cardiovascular Services, Milwaukee, WI
}

Received Nov 29, 2018; accepted Nov 30, 2018

doi:10.1007/s12350-018-01588-6

\section{See related article, pp. 1582-1591}

\section{IN THE BEGINNING}

The application of phase analysis most likely originated with software algorithms used to define the atrioventricular plane in equilibrium radionuclide angiograms (ERNA) for the purpose of calculating the left ventricular ejection fraction. That application was intuitive since the left atrium and left ventricle are $180^{\circ}$ out of phase. Given adequate count statistics to generate reliable Fourier transforms, the technique is quite robust. Early on, the first clinical applications used phase analysis to identify the timing of the onset of mechanical contraction using ERNA in order to characterize electrical phenomena that caused either delays in contraction (LBBB, RBBB) or premature mechanical contraction (site of pre-excitation in WPW, site of origin of ventricular tachycardia). However, despite the elegance of the approach, its application did not gain traction in clinical cardiology. At the time, electrophysiology as a clinical discipline was in its infancy and with its maturation, there was really no need for other noninvasive techniques to demonstrate the location of a Kent bundle or the site of earliest activation during ventricular tachycardia. And so, phase analysis was relegated to the file of curios, something to show trainees or dredge up for an exam.

\footnotetext{
Reprint requests: Steven Port, MD, Advocate Aurora Health, Aurora Cardiovascular Services, Milwaukee, WI; sport@wi.rr.com J Nucl Cardiol 2020;27:1592-5. $1071-3581 / \$ 34.00$

Copyright (C) 2019 American Society of Nuclear Cardiology.
}

\section{CRT}

Fast forward almost a quarter of a century when a clinical problem, left ventricular pump failure, was noted to be exacerbated or caused by electromechanical dyssynchrony in a subset of patients with systolic congestive heart failure, i.e., those with a prolonged QRS duration. Patients with LBBB had the quintessential type of dyssynchrony that was apparent by simply viewing the septal-lateral wall delay on the cinematic display of the left ventricle in either ERNA or gated SPECT MPI or 2D echocardiographic studies. Biventricular pacing was subsequently shown to both restore the synchrony of contraction and, more importantly, improve outcomes. However, not all patients benefitted from resynchronization therapy (CRT). Those with a QRS duration of $>150 \mathrm{~ms}$ seemed to benefit most. Almost overnight, the long-abandoned phase analysis algorithms took center stage along with tissue Doppler and subsequently speckle tracking techniques to quantify electromechanical dyssynchrony in an effort to identify parameters that would best quantify the dyssynchrony and potentially predict which patients would best be served by CRT. In the process of those investigations, it became apparent that dyssynchrony was present and occasionally severe in patients with a normal QRS duration as well. By this time, SPECT MPI had largely replaced ERNA in the assessment of LV function and LV dyssynchrony but not because of its superiority but because of its availability and familiarity.

The lexicon of systolic phase analysis includes the standard deviation of phase angles (PSD), 95\% of the width of the histogram of phase angles in all the pixels in the LV (bandwidth or PBW), and two other rarely used terms, skewness and kurtosis which relate to the distribution of the phase angles in the phase histogram. Normal limits for those values have been published. ${ }^{1}$ Yet, when the wave of activity in quantitative dyssynchrony for CRT patients crested, no generally accepted noninvasive method for selection of patients for CRT emerged. Current ACC/AHA, ESC, and Canadian 
guidelines for selection of patients for CRT therapy give a class I indication to patients on optimal medical therapy with LVEF $<35 \%$, class II-IV CHF, and LBBB or $\mathrm{QRS} \geq 150 \mathrm{~ms}$. No guideline incorporates any echo or radionuclide variable for selection of CRT therapy. Once CRT has been recommended, there may be a role for phase analysis in the proper placement of the LV electrode based on the site of latest activation on the radionuclide study ${ }^{2}$ although that technique has not received widespread acceptance.

While the pursuit of a radionuclide descriptor of dyssynchrony that would be useful in the CRT population was going on, some investigators were also pursuing alternative descriptors of dyssynchrony.

\section{ENTROPY}

The terms synchrony and entropy were first described in the nuclear cardiology literature in $2005 .^{3}$ Both synchrony and entropy outperformed PSD and PBW in distinguishing among several different types of dyssynchrony in a simulation study. Entropy may perform better largely for technical reasons. At its most basic, entropy is a descriptor of randomness in a system. For example, if we think of water, there is a range of entropy of the molecules related to temperature. Entropy decreases when water is cooled and approaches 0 (minimum randomness) when it is completely frozen. As water is heated, molecular movement increases and entropy approaches 1 (maximum randomness) when it is evaporating or boiled. The calculation of systolic phase entropy has some advantages over PSD and PBW because it may better distinguish the dyssynchrony of diffuse abnormalities of depolarization and contraction compared to regional dyssynchrony as is seen with a severe wall motion abnormality or with a conduction abnormality where there are discrete families of altered phase. It is less sensitive to outliers of bandwidth. If we analogize ventricular entropy to the water model described above, the randomness of the electromechanical function of the ventricle increases as its measured entropy increases, not of course due to temperature change but to underlying pathology such as progressive fibrosis and/or ischemia.

\section{BEYOND CRT}

Regardless of the failure to accurately predict success from CRT, echocardiographic studies had shown that mechanical dyssynchrony was common in patients with heart failure, even when the QRS duration was normal and furthermore, dyssynchrony was a significant predictor of outcomes in patients with systolic heart failure. ${ }^{4}$ The prognostic value of systolic PSD and PBW was subsequently demonstrated in patients with end-stage renal disease referred for transplantation evaluation. ${ }^{5}$ More recently, a publication in the Journal ${ }^{6}$ demonstrated that systolic phase entropy was an independent predictor of MACE (sudden cardiac death, fatal arrhythmia, CHF requiring hospitalization) in patients with dilated cardiomyopathy and normal QRS in a multivariable regression analysis including clinical and other radionuclide variables. High- and low-phase entropy were defined as $\geq 0.61$ and $<0.61$ and separated two groups with a significant difference in MACE beginning at 6 months of follow-up and extending to 5 years. Limiting the value of the study was the small population $(n=46)$ and the even smaller number of events including 2 cardiac deaths, 2 resuscitated fatal arrhythmias, and 5 heart failure hospitalizations. Interestingly, the authors of the paper also included myocardial biopsy results with particular attention to the levels of SERCA2a mRNA that codes for a protein involved with calcium transport. Levels of SERCA2a mRNA have been shown to be reduced in heart failure. In this small study, the SERCA2a mRNA level varied inversely with the measurement of phase entropy while LVEF and BNP did not show that relationship. That said, the study should generate more interest in the possible contribution of radionuclide phase analysis of patients with dilated cardiomyopathy beyond the question of CRT.

\section{AND DIASTOLE}

All the previously described efforts in the radionuclide assessment of ventricular dyssynchrony have been focused on systole and the timing of onset of mechanical contraction. But the diastolic portion of the cardiac cycle can also be evaluated using phase analysis. In fact, in two studies using tissue Doppler echo, diastolic dyssynchrony was actually more common than systolic dyssynchrony in patients with CHF. ${ }^{7,8}$ Those findings were subsequently also found to be true in patients with ESRD and normal LVEF when, for the first time, multiharmonic phase analysis was applied to SPECT MPI data. ${ }^{9}$ Both diastolic PSD and PBW (called PHB in the study) were significantly prolonged in the ESRD group compared to controls and diastolic dyssynchrony was present in $65 \%$ of subjects compared to $47 \%$ of subjects with systolic dyssynchrony. Diastolic dyssynchrony was present without systolic dyssynchrony in 21/ 78 patients in the study but when systolic dyssynchrony was present, diastolic dyssynchrony was always present. In the current issue of the Journal, Wang et al. ${ }^{10}$ compare the prognostic significance of systolic and diastolic PSD, PBW, and phase entropy in patients with idiopathic dilated cardiomyopathy (CAD, valvular 
disease, alcoholic or inflammatory disease, or any identifiable systemic illness excluded). They acquired 8-frame SPECT MPI data and processed the data using Emory ERToolbox software. The study used sudden cardiac death, resuscitated fatal arrhythmias, and CHF requiring hospitalization as endpoints. Univariate predictors of events included LVEF, Hypertension, NTproBNP, Systolic PSD, Systolic PBW, Systolic phase entropy, and diastolic PBW. Diastolic phase entropy showed a $P$ value of .08. Multivariable Cox regression analysis using the significant univariate predictors and systolic phase entropy showed the systolic phase entropy to remain significant with a hazard ratio of 3.2. NTproBNP was neutral, LVEF showed minimal impact with a hazard ratio of 0.94 , and hypertension appeared to be protective, being 8 times more common in the survivor group. The authors performed a similar regression analysis with univariate predictors and diastolic phase entropy and found very similar results. Diastolic phase entropy showed a hazard ratio of 3.2 with virtually the same ratios for NT-proBNP, LVEF, and hypertension. What was not available was a multivariable Cox regression that included both systolic phase entropy and diastolic phase entropy. It appears that in the presence of severe LV dysfunction, LV systolic and diastolic dyssynchrony occur with similar frequency although systolic dyssynchrony in the form of phase entropy may be more predictive of outcome than diastolic phase entropy when they coexist.

\section{FUTURE DIRECTIONS}

At this point, the literature on mechanical dyssynchrony includes single-center, predominantly small studies that are largely retrospective. As such, numerous confounders that may affect ventricular function are uncontrolled. A multicenter prospective study with many more patients and more endpoint events should be considered. The effect of the duration of disease on the prevalence of dyssynchrony is unknown. Diastolic LV dysfunction is a consistent part of the aging process but there is little data to demonstrate the effect of age on dyssynchrony because age tends to be clustered in studies looking at congestive heart failure. The effect of medications has not been well studied and needs further investigation. Is the prognostic significance of dyssynchrony the same in patients with a normal QRS compared to those with a wide QRS? The analysis of dyssynchrony is fairly straightforward in both SPECT and ERNA but the reproducibility and accuracy of systolic and diastolic phase data are dependent on adequate count statistics in each pixel of the $\mathrm{LV}$ wall or chamber, respectively. To that end, ERNA can more easily achieve high count statistics. When the question was CRT, most experts in the field favored SPECT MPI because it supplied important information about lateral wall viability that could influence LV pacing as well as data on dyssynchrony. When CRT is not the issue, then ERNA should be reconsidered as a method for investigating dyssynchrony, especially systolic entropy and diastolic entropy. The relationship between systolic and diastolic dyssynchrony remains inadequately defined. It appears that in dilated cardiomyopathy, severe LV dysfunction begets both systolic and diastolic dyssynchrony but do they appear simultaneously or does one beget the other? Do we need disease-specific databases for the different etiologies of systolic heart failure? Does dyssynchrony itself cause LV dysfunction? Subjects with "isolated" LBBB may develop a systolic cardiomyopathy over time. Is there an underlying pathology that causes both the conduction disturbance and the myopathy or is the dyssynchrony the cause of the myopathy? Are there other myopathies that start with or are caused by dyssynchrony? Clinical and echobased diastolic dysfunction is frequently but not always accompanied by diastolic dyssynchrony. What is the difference between those subgroups? Systolic dyssynchrony has also been detected in patients who clinically appear to have pure diastolic dysfunction. Why is that? How does one treat dyssynchrony other than by CRT?

Despite all those questions and concerns, the data now seem consistent enough to believe in the measurements when acquired appropriately and to believe in the prognostic significance of systolic and probably diastolic dyssynchrony. Furthermore, we know that dyssynchrony occurs in the setting of a normal QRS width. Our experience with CRT may have put a damper on the enthusiasm for studying dyssynchrony but there is much to be learned.

\section{Disclosure}

The author has no relevant disclosures.

\section{References}

1. Ji C. Onset of left ventricular mechanical contraction as determined by phase analysis of ECG-gated myocardial perfusion SPECT imaging: Development of a diagnostic tool for assessment of cardiac mechanical dyssynchrony. J Nucl Cardiol 2005;12:68795.

2. Boogers MJ, et al. Optimal left ventricular lead position assess with phase analysis on gated myocardial perfusion SPECT. Eur J Nucl Med Mol Imaging 2011;38:230-8.

3. O'Connell JW, et al. A unique method by which to quantitate synchrony with equilibrium radionuclide angiography. J Nucl Cardiol 2005;12:441-50.

4. Cho GY, et al. Mechanical dyssynchrony assess by tissue Doppler imaging is a powerful predictor of mortality in congestive heart 
failure with normal QRS duration. J Am Coll Cardiol 2005;46:2237-43.

5. Aljaroudi, et al. Impact of left ventricular dyssynchrony on outcomes in patients with end stage renal disease. J Nucl Cardiol 2010;17:1058-64.

6. Kano N, et al. Left ventricular phase entropy: Novel prognostic predictor in patients with dilated cardiomyopathy and narrow QRS. J Nucl Cardiol 2018;25:1677-87.

7. Schuster I, et al. Diastolic asynchrony is more frequent than systolic asynchrony in dilated cardiomyopathy and is less improved by cardiac resynchronization therapy. J Am Coll Cardiol 2005;46:2250-7.

8. Wang J, et al. Systolic and diastolic dyssynchrony in patients with diastolic heart failure and the effect of medical therapy. J Am Coll Cardiol 2007;49:88-96.
9. Chen J, et al. Left-ventricular systolic and diastolic dyssynchrony as assessed by multi-harmonic phase analysis of gated SPECT myocardial perfusion imaging in patients with end-stage renal disease and normal LVEF. J Nucl Cardiol 2011;18:299-308.

10. Wang $\mathrm{C}$ et al. Prognostic value of left ventricular systolic and diastolic dyssynchrony measured from gated SPECT MPI in patients with dilated cardiomyopathy. J Nucl Cardiol. https://doi. org/10.1007/s12350-018-01468-z.

Publisher's Note Springer Nature remains neutral with regard to jurisdictional claims in published maps and institutional affiliations. 